polarization causes $Q_{\pi \sigma}$ partly to cancel $^{24} Q_{\pi \sigma^{\prime}}$ and so to reduce the calculated MO approximation $D_{N Q}$, making it agree better with the observed value. ${ }^{25}$ This improvement most probably much outweighs the contrary effects ${ }^{22 a}$ obtained by optimizing $Z^{*}$.

The effect on $D_{N Q}$ of using polarized AO's is quite different in the AO than in the MO approximation. Referring to an earlier paragraph on the variation of $Z^{*}$ in the $\mathrm{AO}$ approximation,

\footnotetext{
${ }^{24}$ Presumably $Q_{\pi \sigma}$ does not become so large as to more than cancel $Q_{\pi \sigma^{*}}$, but this cannot be shown by the present qualitative arguments.

${ }_{25}$ This applies to the heavier halogens. For $F_{2}$ the result is not qualitatively obvious, but presumably still holds, since the best-MO approximation pretty surely should give better $D_{N Q}$ values than the LCAO approximation.
}

similar remarks now apply for improvement of the AO's by polarization. The only AO's for which improvement is important are the $n p \sigma$ AO's in state $Q$. These are occupied by electrons which are somewhat bonding, so to minimize the energy the polarization should be such as to give increased overlapping. This would be obtained by using Eq. (35) with $l / k$ positive, instead of negative as for best polarization of the LCAO MO's.

The effects of such polarization on $Q_{N Q}$ can be seen from Eq. (32). $S_{\sigma \sigma^{\prime}}$ and $Q_{\pi \sigma^{\prime}}$ are increased, while $Q_{\pi \sigma}$ takes on a negative value, so that $D_{N Q}$ is increased. Agreement with experiment is made worse, opposing ${ }^{22 a}$ the effect obtained by optimizing $Z^{*}$.

\title{
The Hydrogen Isotope Exchange Reaction
}

\author{
J. O. HALFORD \\ Department of Chemistry, University of Michigan, Ann Arbor, Michigan
}

(Received August 25, 1939)

\begin{abstract}
The possibility of deriving the equilibrium constants for the higher deuterium substitutions from the isotope exchange coefficient is examined with the aid of calculations on the ammonia and methane reactions with water. If the experiment is carried to solutions at equilibrium with water containing as much as 25 percent $\mathrm{D}_{2} \mathrm{O}$ and the error in a single measurement does not exceed 1.0 percent the second constant is determined to within 10 percent of the correct value. As a standard of comparison against which the experimental results may be examined to advantage, an equation is developed showing the relation between the equilibrium constant for the first substitution and the exchange factor at higher deuterium fractions, subject to the assumption that each successive substitution of $D$ for $H$ produces the same frequency shift. The evaluation of frequency shifts from exchange data is considered.
\end{abstract}

$T^{\prime}$ HE study of hydrogen isotope exchange equilibria has been restricted almost exclusively to systems of low deuterium fraction in which only a negligible proportion of the molecules can acquire more than one deuterium atom. For exchange reactions involving water this restriction limits the interpretation to the first of the $n$ reactions

$$
A \mathrm{H}_{n}+p \mathrm{DOH}=A \mathrm{H}_{n-p} \mathrm{D}_{p}+p \mathrm{HOH} .
$$

In principle the measurement and interpretation need not be so limited. It is possible to perform exchange experiments over a wide range of isotope fractions involving systems in which the higher deuterium substitution products are pres- ent at considerable concentrations. From such experiments, if they were sufficiently accurate, all the equilibrium constants of the reactions expressed by Eq. (1) could be derived. The success of an actual interpretation, however, is limited by the accuracy of the individual distribution measurement and by the fact that the relative scarcity of deuterium makes it impractical to obtain data over the entire range of isotope compositions.

In the following paragraphs it is shown by calculations with the ammonia and methane reactions that the constant for the second substitution can be obtained with a fair degree of accuracy even with a liberal allowance for experi- 
mental error and that the satisfactory determination of the higher constants would involve a greater precision than is normally achieved. The equilibrium constants are derived from the distribution by trial. The extrapolation to zero deuterium fraction for $K_{1}$ and the selection of first approximations to $K_{2}$ and $K_{3}$ are facilitated by the use of some limiting algebraic relations. For their development it is convenient to define $\beta_{A}$ by the equation

$$
\beta_{A}=\sum_{p=1}^{p=n} p A_{p} / \sum_{p=0}^{p=n} A_{p},
$$

in which $A_{p}$ represents the number of molecules of $A \mathrm{H}_{n-p} \mathrm{D}_{p}$ in the equilibrium mixture. Eq. (2) then describes the exchange experiment in terms of the average number of $\mathrm{D}$ atoms per molecule of $A \mathrm{H}_{n}$ and its deuterium substitution products.

Each substance $A \mathrm{H}_{n-p} \mathrm{D}_{p}$ will come to equilibrium with $\mathrm{H}_{2} \mathrm{O}, \mathrm{DOH}$ and $A \mathrm{H}_{n}$ in accordance with reaction 1 for which the mass law expression is

$$
A_{p}=A_{0} K_{p} R^{p} .
$$

The concentration ratio $\mathrm{DOH} / \mathrm{HOH}$ has been represented by $R$. Eqs. (2) and (3) are combined to yield the relation

$$
\beta_{A}=\sum_{p=1}^{p=n} p K_{p} R^{p} / 1+\sum_{p=1}^{p=n} K_{p} R^{p}
$$

The corresponding expression for $\beta_{\mathrm{H}_{2}} \mathrm{O}$ takes the form

$$
\beta_{\mathrm{H}_{2} \mathrm{O}}=R(1+2 K R) /\left(1+R+K R^{2}\right),
$$

with $K$ denoting the equilibrium constant of the reaction

$$
2 \mathrm{DOH}=\mathrm{H}_{2} \mathrm{O}+\mathrm{D}_{2} \mathrm{O} \text {. }
$$

In order to develop Eq. (4) any further it is necessary to assume relations between the $n$ constants $K_{p}$. The numerical or symmetry effect can be separated from the other statistical factors by means of the equation

$$
K_{p}=\frac{k_{p}}{2^{p}} \frac{n !}{(n-p) ! p !} .
$$

If $k_{p}$ is set equal to $k_{1}{ }^{p}$ it follows that

$$
K_{p}=\left(\frac{K_{1}{ }^{\prime}}{n}\right)^{p} \frac{n !}{(n-p) ! p !}
$$

Eq. (8) describes the limiting case for which the same factors of mass, moment of inertia and frequency could be used to calculate the equilibrium constant of each successive reaction in the series

$$
A \mathrm{H}_{n-p} \mathrm{D}_{p}+\mathrm{DOH}=A \mathrm{H}_{n-p-1} \mathrm{D}_{p+1}+\mathrm{H}_{2} \mathrm{O} .
$$

This condition is fulfilled closely enough by the mass and inertia factors but may be far from true for the vibrational effect. Consequently the derivation leads to a relation in which $K_{1}{ }^{\prime}$ varies with $\beta_{A}$ in a manner determined primarily by the change in frequency shift with successive substitution of $\mathrm{D}$ for $\mathrm{H}$.

Equation (8) is equivalent to a conclusion reached by Brodskii. ${ }^{1}$ It is combined with Eq. (4) and simplified as follows:

$$
\beta_{A}=\frac{\sum_{p=1}^{p=n}\left(\frac{K_{1}{ }^{\prime} R}{n}\right)^{p} \frac{n !}{(n-p) !(p-1) !}}{1+\sum_{p=1}^{p=n}\left(\frac{K_{1}{ }^{\prime} R}{n}\right)^{p} \frac{n !}{(n-p) ! p !}}=\frac{n K_{1}{ }^{p} R}{n+K_{1}{ }^{\prime} R} .
$$

It follows that

$$
K_{1}^{\prime}=n \beta_{A} / R\left(n-\beta_{A}\right)
$$

for the limiting case under consideration.

The numerical factor introduced into Eq. (7) requires no justification, since it merely sets the probability of occurrence of $A \mathrm{H}_{n-p} \mathrm{D}_{p}$ proportional to the number of permutations of $(n-p)$ atoms of the one isotope with $p$ atoms of the other. It is of interest, however, to consider the relation of this factor to the symmetry number which occurs in the simplified form of the partition function or sum over states.

In the general case, $A \mathrm{H}_{n-p} \mathrm{D}_{p}$ may represent several molecules, the number depending on the symmetry number $\sigma_{0}$ of $A \mathrm{H}_{n}$. To the $i$ th molecule is assigned the symmetry number $\sigma_{p i}$. The constant $K_{p i}$ of the reaction

$$
A \mathrm{H}_{n}+p \mathrm{DOH}=\left(A \mathrm{H}_{n-p} \mathrm{D}_{p}\right)_{i}+p \mathrm{H}_{2} \mathrm{O}
$$

then becomes

$$
K_{p i}=\left(k_{p i} / 2^{p}\right)\left(\sigma_{0} / \sigma_{p i}\right) .
$$

The relations equivalent to Eqs. (7) and (8) are obtained by summing over the $i$ molecules:

$$
K_{p}=\sum_{i} K_{p i}=\frac{1}{2^{p}} \sum_{i} k_{p i} \underset{\sigma_{p i}}{\sigma_{0}}=\frac{k_{p}}{2^{p}} \sum_{i} \frac{\sigma_{0}}{\sigma_{p i}} .
$$

\footnotetext{
${ }^{1}$ Brodskii, Trans. Faraday Soc. 33, 1180 (1937).
} 
TABLE I. Statistical constants for ammonia-water and methane-water exchange reactions at $25^{\circ} \mathrm{C}$.

\begin{tabular}{|c|c|c|c|c|c|}
\hline & $\left(\frac{M_{p}}{M_{n}}\right)^{\frac{1}{2}}$ & $\left(\frac{A_{p} B_{p} C_{p}}{A_{0} B_{0} C_{0}}\right)^{\frac{1}{2}}$ & $-\Delta \nu\left(\mathrm{CM}^{-1}\right)$ & $K_{p}$ & $\begin{array}{l}\left(\frac{K_{1}}{n}\right)^{p} \\
\frac{n !}{(n-p) ! p !}\end{array}$ \\
\hline $\begin{array}{l}\mathrm{H}_{2} \mathrm{O} \\
\mathrm{DOH} \\
\mathrm{D}_{2} \mathrm{O}\end{array}$ & $\begin{array}{l}1.000 \\
1.085 \\
1.172\end{array}$ & $\begin{array}{l}1.000 \\
1.659 \\
2.632\end{array}$ & $\begin{array}{c}0 \\
1133^{\Delta} \\
2377^{\Delta}\end{array}$ & E & E \\
\hline $\begin{array}{l}\mathrm{NH}_{3} \\
\mathrm{NH}_{2} \mathrm{D} \\
\mathrm{NHD}_{2} \\
\mathrm{ND}_{3}\end{array}$ & $\begin{array}{l}1.000 \\
1.090 \\
1.183 \\
1.278\end{array}$ & $\begin{array}{l}1.000 \\
1.457 \\
2.043 \\
2.752\end{array}$ & $\begin{array}{c}0 \\
1097^{*} \\
2417^{*} \\
3850^{*}\end{array}$ & $\begin{array}{l}\overline{1.214} \\
0.804 \\
0.223\end{array}$ & $\begin{array}{l}\overline{1.214} \\
0.491 \\
0.066\end{array}$ \\
\hline $\begin{array}{l}\mathrm{CH}_{4} \\
\mathrm{CH}_{3} \mathrm{D} \\
\mathrm{CH}_{2} \mathrm{D}_{2} \\
\mathrm{CHD}_{3} \\
\mathrm{CD}_{4}\end{array}$ & $\begin{array}{l}1.000 \\
1.096 \\
1.194 \\
1.296 \\
1.400\end{array}$ & $\begin{array}{l}1.000 \\
1.342 \\
1.776 \\
2.269 \\
2.827\end{array}$ & $\begin{array}{c}0 \\
1161 \ddagger \\
2441 \ddagger \\
3725 \ddagger \\
5004 \ddagger\end{array}$ & $\begin{array}{l}\overline{1.747} \\
1.495 \\
0.553 \\
0.073\end{array}$ & $\begin{array}{l}\overline{1.747} \\
1.145 \\
0.333 \\
0.036\end{array}$ \\
\hline
\end{tabular}

$\triangle$ Barker and Sleator, J. Chem. Phys. 3, 660 (1935).

* Howard, J. Chem. Phys. 3, 208 (1935)

$\neq$ Benedict, Morikawa, Barnes and Taylor, J. Chem. Phys. 5, 1

TABLE II. Ammonia exchange reaction at $25^{\circ} \mathrm{C}$. Distribution from molecular constants.

\begin{tabular}{cccc}
\hline$R$ & \% $_{2} \mathrm{O}$ & $\beta$ & $K_{1^{\prime}}$ \\
\hline 0.6 & 24.09 & $0.702_{7}$ & $1.529_{4}$ \\
.4 & 17.22 & $.482_{5}$ & $1.437_{3}$ \\
.3 & 13.40 & $.365_{3}$ & $1.386_{5}$ \\
.2 & 9.27 & $.244_{7}$ & $1.332_{3}$ \\
.1 & 4.81 & $.122_{3}$ & $1.274_{8}$ \\
.05 & 2.45 & .0610 & $1.244_{9}$ \\
0 & 0 & 0 & 1.214 \\
\hline
\end{tabular}

The factor $k_{p}$ is a weighted average of the values of $k_{p i}$. If both Eqs. (7) and (13) are correct it is necessary to set $\sum_{i} \sigma_{0} / \sigma_{p i}$ equal to $n ! /(n-p) ! p !$

In the limiting case for which $\sigma_{0}=1$ each $\sigma_{p i}$ must be equal to unity because each substitution of $\mathrm{D}$ for $\mathrm{H}$ will result in a recognizably different molecule. The ratio $\sigma_{0} / \sigma_{p i}$ is equal to unity and the sum over the $i$ forms will be equal to the number of forms $n ! /(n-p) ! p !$, that is, to the number of distinguishable permutations of $p \mathrm{D}$ atoms with $(n-p) \mathrm{H}$ atoms. If, however, $\sigma_{0}$ equals $a b c$, the product of a set of numbers representing equivalent arrangements produced by internal rotation or rotation of the entire molecule, the substitution of $p \mathrm{D}$ atoms will eliminate one or more of these factors, so that $\sigma_{p i}$ might be equal to $a$ and $\sigma_{0} / \sigma_{p i}$ equal to $b c$. The rotational operations corresponding to $b$ and $c$, when performed upon the substitution product, are no longer symmetry operations, but instead show the equivalence of $b c$ of the total possible permutations. In this way they give to the form of symmetry number $a$ a statistical weight equal to the number of permutations which can be shown to be equivalent by performing just those rotations which were eliminated as symmetry operations by the substitution. None of the $n ! /(n-p) ! p$ ! permutations are lost and each one must belong to one of the $i$ forms. The quantity $\sum_{i} \sigma_{0} / \sigma_{p i}$ must therefore be independent of the actual symmetry numbers and equal to the number of permutations. It can be shown, in addition, that this relation is a necessary consequence of the accepted principle that the difference between isotopes of slightly different mass, such as $\mathrm{Cl}^{35}$ and $\mathrm{Cl}^{37}$, may be taken into account or neglected at will without introducing significant statistical errors.

For the purpose of illustrating the sensitivity of $K_{2}$ and $K_{3}$ to variations in the distribution values from which they have been calculated, it has seemed preferable to use the ammonia and methane reactions, rather than to set up a purely hypothetical case. All molecular data necessary to a complete description of these systems are available. Table I summarizes the values of $K_{p}$ at $25^{\circ} \mathrm{C}$ together with the mass ratios, moment ratios and frequencies used in their calculation. $K_{p}$ has been obtained from the relation $K_{p}=L_{p} / L_{\mathrm{DOH}^{p}}{ }^{p}$ where

$L_{p}=\left(\frac{M_{p}}{M_{0}}\right)^{\frac{3}{3}}\left(\frac{A_{p} B_{p} C_{p}}{A_{0} B_{0} C_{0}}\right)^{\frac{1}{2}} e^{-h c \Delta p / 2 k T} \frac{n !}{(n-p) ! p !}$

Factors of the form $\left(1-e^{-h c \nu / k T^{\prime}}\right)$, associated with individual frequencies, have been omitted from Eq. (14) because their contribution to $K_{p}$ is negligible. The moment ratios are based upon the assumption that interatomic distances and bond angles do not change with the isotope substitution. The tetrahedral angle has been used for methane, and for water and ammonia, respectively, the angles taken are $105^{\circ}$ and $113^{\circ}$. It is possible that some of the frequency shifts may be in error, since they are based in part upon calculated quantities. For example, the data of Ginsburg and Barker ${ }^{2}$ set the first methane shift at 1088, which would give a constant about

\footnotetext{
${ }^{2}$ Ginsburg and Barker, J. Chem. Phys. 3, 668 (1935).
} 
TABLE III. Methane exchange reaction at $25^{\circ} \mathrm{C}$. Distribution from molecular constants.

\begin{tabular}{cccc}
\hline$R$ & $\% \mathrm{D}_{2} \mathrm{O}$ & $\beta$ & $K_{\mathbf{1}^{\prime}}$ \\
\hline 0.6 & 24.09 & $0.928_{4}$ & $2.014_{8}$ \\
.4 & 17.22 & $.653_{5}$ & $1.952_{8}$ \\
.3 & 13.40 & $.502_{0}$ & $1.913_{3}$ \\
.2 & 9.27 & $.346_{5}$ & $1.866_{6}$ \\
.1 & 4.81 & $.173_{3}$ & $1.811_{7}$ \\
.05 & 2.45 & .0871 & $1.780_{8}$ \\
0 & 0 & 0 & 1.747 \\
\hline
\end{tabular}

15 percent lower than the value of 1.747 which appears in the table. This difference is not important for the present purpose.

The constants of Table I are used to yield values of $\beta$, in accordance with $\mathrm{Eq}$. (5), for selected values of $R$. These quantities, together with $K_{1}$ from Eq. (10) and, for reference, the deuterium oxide concentration of the equilibrium water in the conventional units, are presented in Tables II and III, respectively, for the ammonia and methane reactions.

The practical experimental range has been taken to involve equilibration with water containing not more than 25 percent $\mathrm{D}_{2} \mathrm{O}$, although for highest accuracy in determining the constants $K_{p}$ the distribution should cover the entire range of isotope fractions, and each constant should be derived mainly from the distribution in the region in which $A \mathrm{H}_{n-p} \mathrm{D}_{p}$ contains its maximum fraction of the deuterium. Tables II and III show the character to be expected for typical isotope exchange data involving small molecules in which the replaceable hydrogens are linked to a single atom. Such data could be conveniently extrapolated to zero deuterium fraction to yield the limiting value of $K_{1}$ by plotting both $K_{1}{ }^{\prime}$ and $\beta / R$ against $R$ and taking the best intercept common to the two functions. For selecting a preliminary value of $K_{2}$ from experimental data, a comparison of $K_{1}{ }^{\prime}$ with the quantities appearing in Tables I, II and III should prove to be useful. In this connection, it is observed that the deviation of $K_{1}{ }^{\prime}$ from $K_{1}$ at a given value of $R$ is about the same for the ammonia and methane reactions, in spite of the fact that $K_{2}$ is much closer to $K_{1}^{2} / n$ in the latter case. This indicates that the deviation of $K_{1}$ from constancy is larger for larger values of $n$, other factors being constant.

In testing the sensitivity of $K_{2}$ to uncertainties in the data from which it is derived, it is assumed that each experimental value of $\beta$ will be accurate to 1.0 percent, and that $\beta$ values used in the interpretation will be taken from a smooth curve of $\beta$ against $R$, so that the sum of the errors in two $\beta$ values separated by 0.3 unit on the $R$ scale will not exceed 1.0 percent. In Tables IV and $V$ are shown $K_{2}$ and $K_{3}$ as derived from $K_{1}$ and values of $\beta$ at $R=0.3$ and $R=0.6$ with these quantities differing from those of Tables II and III in the manner indicated in the first three columns. The fourth and sixth columns show $K_{2}$ and $K_{3}$, the fifth and seventh the corresponding frequency shifts when the entire deviation from Eq. (8) is assumed to be due to the frequencies. This assumption introduces an error of $18 \mathrm{~cm}^{-1}$ in $\Delta \nu_{2}$ for ammonia and would have a much smaller effect in the interpretation of data for larger molecules.

The largest tabulated deviation of $K_{2}$ from the correct value is 8.2 percent. The corresponding error in $\Delta \nu_{2}$ is $53 \mathrm{~cm}^{-1}$, of which $18 \mathrm{~cm}^{-1}$ could be accounted for in a more detailed calculation. It is interesting to observe that this is considerably smaller than the difference of $73 \mathrm{~cm}^{-1}$ between the calculated and observed values of $\Delta \nu_{1}$ for methane to which reference was made in connection with Table I. It is evident that the determination of $K_{2}$ and $\Delta \nu_{2}$ from exchange equilibrium data of reasonable accuracy is feasible, but that satisfactory evaluation of the corresponding quantities for the third substitution would require measurements of higher precision extending over a wider range of isotope fractions. Exchange data can thus provide an additional condition which must be met by an assignment of frequencies to a series of molecules $A \mathrm{H}_{n-p} \mathrm{D}_{p}$.

There are no recorded exchange experiments

TABLE IV. Ammonia exchange reaction at $25^{\circ} \mathrm{C}$; Variation of $K_{2}$ and $K_{3}$ with $K_{1}$ and $\beta$.

\begin{tabular}{c|c|c|c|c|c|c}
\hline \multicolumn{2}{c|}{$1.0 \%$ VARIATION } & & & & \\
\cline { 1 - 2 }$K_{1}$ & $\beta(0.3)$ & $\beta(0.6)$ & $K_{2}$ & $\Delta \nu_{2}(2417)$ & $K_{3}$ & $\Delta v_{3}(3850)$ \\
\hline 0 & 0 & 0 & 0.804 & 2399 & 0.223 & 3796 \\
0 & - & 0 & .738 & 2364 & .285 & 3899 \\
0 & - & - & .763 & 2377 & .233 & 3815 \\
- & $\mathbf{0}$ & - & .855 & 2424 & .150 & 3632 \\
- & 0 & + & .805 & 2400 & .255 & 3853 \\
\hline
\end{tabular}


with any substance for which $n>1$ which extend over a sufficient range of isotope fractions to permit an accurate interpretation along the lines illustrated above. Some results ${ }^{3}$ obtained in this laboratory with acetone, however, may be examined with profit in spite of their inadequacy. They are shown in Table VI.

From the last four listed measurements it appears that the limiting $K_{1}$ at $25^{\circ} \mathrm{C}$ should be about 2.45 , giving 0.82 for the exchange factor. This is in very close agreement with the reciprocal of Brodskii's exchange factor of 1.22 and agrees well with the calculated result of $\mathrm{K}$. Wirtz. ${ }^{4}$ A factor for internal rotation or torsional vibration of the methyl group, equal in the first approximation to $(4 / 3)^{\frac{1}{3}}$, is introduced into $\mathrm{Eq}$. (14), and $\Delta \nu_{1}$ is calculated to be $-1217 \mathrm{~cm}^{-1}$. This method of taking internal rotation into account, which has been used by Hobden, Johnston, Weldon and Wilson, ${ }^{5}$ by Halford and

TABLE V. Methane exchange reaction at $25^{\circ} \mathrm{C} ;$ Variation of $K_{2}$ and $K_{3}$ with $K_{1}$ and $\beta$.

\begin{tabular}{|c|c|c|c|c|c|c|}
\hline \multicolumn{3}{|c|}{$1.0 \%$ VARIaTION } & \multirow[b]{2}{*}{$K_{2}$} & \multirow[b]{2}{*}{$\Delta \nu_{2}(2441)$} & \multirow[b]{2}{*}{$K_{3}$} & \multirow[b]{2}{*}{$\Delta \nu_{3}(3725)$} \\
\hline$K_{1}$ & $\boldsymbol{\beta}(0.3)$ & $\beta(0.6)$ & & & & \\
\hline 0 & 0 & 0 & 1.495 & 2433 & 0.553 & 3694 \\
\hline 0 & + & 0 & 1.605 & 2461 & .458 & 3615 \\
\hline 0 & + & + & 1.555 & 2450 & .558 & 3698 \\
\hline+ & 0 & + & 1.412 & 2409 & .680 & 3780 \\
\hline+ & 0 & - & 1.514 & 2438 & .479 & 3634 \\
\hline
\end{tabular}

${ }^{3}$ Halford, Anderson, Bates and Swisher, J. Am. Chem. Soc. 57, 1663 (1935).

${ }^{4}$ Wirtz, Zeits. f. physik. Chemie, B31, 309 (1936).

'Hobden, Johnston, Weldon and Wilson, J. Chem. Soc. 61 (1939).
TABLE VI. Acetone exchange reaction.

\begin{tabular}{c|c|c|c|c}
\hline \hline TEMP. $\left({ }^{\circ} \mathrm{C}\right)$ & $\% \mathrm{D}_{2} \mathrm{O}$ & \multicolumn{1}{|c|}{$R$} & $\boldsymbol{\beta}$ & \multicolumn{1}{|c|}{$K_{\mathrm{1}^{\prime}}$} \\
\hline 80 & 5.17 & 0.1080 & 0.2789 & 2.71 \\
80 & 4.14 & .0857 & .2153 & 2.61 \\
80 & 1.89 & .0384 & .0860 & 2.27 \\
$80^{*}$ & 0.919 & .01852 & .0400 & 2.17 \\
60 & 1.136 & .02293 & .0524 & 2.31 \\
40,35 & 1.167 & .02356 & .0561 & 2.40 \\
\hline
\end{tabular}

* Reversed reaction, subject to larger experimental error.

Pecherer, ${ }^{6}$ and by Wirtz, is equivalent to an assumption that the contribution of this type of motion to the exchange equilibrium constant is not sensitive to the height of the potential barrier. The frequency shift determines the temperature dependence of the equilibrium constant and permits the calculation of $K_{1}$ at $80^{\circ} \mathrm{C}$ to be 2.37 , a value considerably higher than the experimental one at this temperature. The calculated value is taken to be the more reliable and is combined with the first two measurements of Table VI to yield $K_{2}=4.1$ and $\Delta \nu_{2}=-2650 \mathrm{~cm}^{-1}$. These are minimum magnitudes consistent with available information.

With this interpretation the acetone reaction falls at least qualitatively into line with other exchange reactions. The frequency shift produced by single substitution in the methyl group is numerically greater than $\Delta \nu_{1}$ for methane by an amount comparable to the parallel difference observed between the alcohols and water. In addition, the relation between the indicated values of $\Delta \nu_{1}$ and $\Delta \nu_{2}$ for acetone is similar to that observed for water, ammonia and methane.

${ }^{6}$ Halford and Pecherer, J. Chem. Phys. 6, 571 (1938) 